\title{
Testing the Effectiveness of Cognitive Behavioral Therapy in Relieving Nurses' Ageism Toward Older Adults: A Randomized Controlled Trial
}

\author{
Mohammad Rababa ${ }^{1}$ [ $\cdot$ Hossam Alhawatmeh ${ }^{1} \cdot$ Nahla Al Ali $^{2} \cdot$ Manal Kassab $^{3}$
}

Accepted: 13 October 2020 / Published online: 20 October 2020

(c) Springer Science+Business Media, LLC, part of Springer Nature 2020

\begin{abstract}
Background Although cognitive-behavioral therapy (CBT) techniques are well known for targeting psychological distresses, to date, no study has investigated their effectiveness in relieving death anxiety and ageism among nurses.

Methods A parallel randomized controlled trial was conducted according to the CONSORT guidelines during October 2019 at the university hospital. A total of 110 nurses were selected through proportional stratified sampling and randomly assigned to the experimental and control groups. The intervention consisted of six two-hour training sessions delivered over five modules with the integration of different CBT exercises. The effect of CBT was assessed by measuring the differences in the students' responses to a series of validated questionnaires of study variables pre-test (before the training sessions) and post-test (after the training sessions). Clinical registration was completed at ClinicalTrial.gov (ID: NCT04319393).

Results Overall, using CBT techniques led to significant improvements in the study outcomes. At postintervention, the nurses who had received CBT training had significantly better self-esteem and interpersonal relationships, a greater sense of symbolic immortality, and less death anxiety and ageism than the nurses in the control group.

Conclusions CBT is an effective evidence-based psychological intervention for relieving nurses' ageism towards older adults. The psychological well-being of nurses caring for older adults is crucial, and new techniques should be adopted to relieve nurses' accumulated stress and decrease their death anxiety. Future studies which investigate the effectiveness of CBT on other forms of discrimination, such as racism and sexism in healthcare settings, are recommended.
\end{abstract}

Keywords Ageism $\cdot$ Cognitive-behavioral therapy $\cdot$ Death anxiety $\cdot$ Older adults $\cdot$ Randomized controlled trial

Electronic supplementary material The online version of this article (https://doi.org/10.1007/s10608-020-10167-4) contains supplementary material, which is available to authorized users.

Mohammad Rababa

mjrababa@just.edu.jo

1 Department of Adult Health Nursing, Faculty of Nursing, Jordan University of Science and Technology/School of Nursing, P O Box 3030, Irbid 22110, Jordan

2 Department of Community and Psychiatric Health Nursing, Faculty of Nursing, Jordan University of Science and Technology, Irbid, Jordan

3 Department of Maternal and Child Health Nursing, Faculty of Nursing, Jordan University of Science and Technology, Irbid, Jordan

\section{Introduction}

Ageism is defined as "aged-based discrimination, similar to discrimination on the ground of sex (sexism) or ethnicity (racism)" (Shiovitz-Ezra et al. 2016, p. 299). Although ageism may occur at both structural and individual levels, its impact is underestimated (Chang et al. 2020). Ageism is highly prevalent in healthcare settings among different groups of health care providers and particularly among nurses, who frequently provide close care to older adults (Wyman et al. 2018). To illustrate, in a National Opinion Polls (NOP) survey, $16 \%$ of the interviewed nurses reported that older adults are made to wait longer than younger adults for national health service treatment, and 33\% reported that older adults do not receive the same quality of hospital care received by younger adults (Glendenning 2018). According to a recent study, $72 \%$ of hospitalized older adults in the US and $68 \%$ in Canada have been victims of ageist acts by nurses during their hospitalization (Ben-Harush et al. 2017). 
In the Middle East, $78 \%$ of Jordanian nurses reported at least one ageist opinion or act towards older adults (Rababa et al. 2020).

Ageism in healthcare settings may take many forms, including the unfair allocation of healthcare resources and services and physical or verbal abuse (Wyman et al. 2018). Ageism is associated with negative stereotypes about older adults. The negative stereotypes about older adults held by some nurses may lead them to believe that older adults are frail, weak, dependent, depressed, sick, cognitively dis-intact, and non-productive or lazy. Also, older adults, particularly due to their atypical presentation of illness, are sometimes viewed by nurses as being very challenging patients to care for, deal with, or understand (Rababa et al. 2020). Therefore, due to their frequent exposure to frail older adults, nurses are more likely than other groups of healthcare providers to hold ageist stereotypes (Kessler et al. 2014).

Ageism negatively affects older adults' health-related quality of life and the quality of health care they receive. It also has many negative psychological and physiological health consequences on older adults (Chang et al. 2020). The psychological consequences include the sense of loss of independence and self-control, social isolation, disengagement from life, depression, low self-esteem and autonomy, compromised decision-making abilities, shame, self-hatred, and, in extreme cases, suicide (São José et al. 2019). The physiological consequences include adverse cardiovascular outcomes, increased functional and cognitive disability, pressure ulcers, iatrogenic adverse outcomes, and increased mortality rates (São José et al. 2019).

People over the age of 65 are the fastest-growing age group and the biggest consumer of health care services and resources, particularly in the case of older adults in their final years of life (Salter and Salter 2018). It is commonly believed by healthcare providers that limited healthcare resources should be prioritized for younger adults, who are believed to be more worthy than older adults of living (Gipe and Kerstein 2018). This ageist belief leads to the unfair allocation of healthcare resources between different age groups of patients, which may result in older adults not receiving the healthcare services they need (Krütli et al. 2016). Therefore, many older adults may die with unmet physical and psychosocial needs.

Palliative care for frail older adults is regarded by many healthcare providers as undesirable and useless. According to Fialová et al. (2018), the comfort needs of older adults are frequently unmet, and they are prescribed fewer analgesics than other patients due to nurses viewing them as incurable patients. Many healthcare providers, including nurses, admit to feeling a lack of emotional engagement when caring for frail older adults in intensive care units (Cohen-Mansfield et al. 2019; Kessler et al. 2014). Further, there is a wide prevalence of disrespect towards older adults across different health care settings. This disrespect is perceived as being a result of cultural bias towards an ideal of youth, productivity, and independence (Levy 2018). This cultural bias puts older adults at a medical disadvantage compared to younger patients (Levy 2018).

One explanation for the ageist attitudes of nurses may be the death anxiety experienced by nurses who work with older adults. According to terror management theory (TMT), death anxiety is a dominant-negative emotion experienced by people who constantly think about mortality salience, which is the state of realizing the inevitability of one's death (Helm et al. 2018). Therefore, older adults serve as a potent reminder of mortality salience for nurses, which provokes nurses' prosocial behaviors towards their in-group members (nurses) and ageist behaviors towards their out-group members (older adults) (Helm et al. 2018). This hypothesis has been supported by the findings of recent studies, which have indicated a significant association between ageism and death anxiety (Barnett and Adams 2018; Bergman et al. 2018). For example, based on three experiments, Bergman et al. (2018) found death anxiety to be a predictor of ageism in undergraduate students.

TMT also hypothesizes that people have an anxiety buffering system consisting of three components: symbolic immortality, self-esteem, and interpersonal relationships (Helm et al. 2018). This buffering system enables people to cope with their unconscious awareness of their eventual mortality. According to TMT, symbolic immortality is defined as a common shared vision of how people with a similar cultural background view and interact with mortality salience, whilst self-esteem refers to the extent to which one is satisfied with one's own life in light of the standards of life as perceived by people worldwide. Meanwhile, interpersonal relationships are defined by TMT as a set of socially valued attributes that make a person more attractive and likable by others (Helm et al. 2018). It is suggested that enhancing these three components in nurses provides them with a sense of security and safety that counteracts feelings of death anxiety and, in turn, reduces ageism against older adults (Ben-Harush et al. 2017).

In order to strengthen nurses' anxiety buffering systems and thus change their ageist attitudes and behaviors, psychological interventions, such as the implementation of (CBT) techniques, are recommended. CBT techniques are evidencebased psychological interventions widely used for promoting mental health (Dobson and Dobson 2018). CBT focuses on improving the personal coping strategies used for changing negative thoughts and emotions and is currently used for targeting a wide range of mental conditions, including different kinds of anxiety (Dobson and Dobson 2018). A variety of CBT techniques have been suggested for relieving death anxiety, including cognitive restructuring, graded exposure, mindfulness meditation, interpersonal skills training, and 
activity scheduling (Menzies and Dar-Nimrod 2017). Several recent studies have confirmed the effectiveness of CBT in strengthening the structure of anxiety buffering systems by fostering self-esteem (Kunikata et al. 2016), interpersonal relationships (Song et al. 2018), and symbolic immortality (Kissane et al. 2004).

Despite this, no study to date has investigated the effectiveness of the application of CBT techniques in reducing nurses' levels of death anxiety and ageism towards older adults. Accordingly, the current study aimed to test the effectiveness of CBT techniques in reducing nurses' death anxiety and, in turn, eliminating their ageist attitudes toward older adults. Therefore, the study was guided by the following question: Are there significant differences in the nurses' levels of self-esteem, interpersonal relationships, symbolic immortality, death anxiety, and ageism between the control group and the treatment group?

\section{Method}

\section{Design, Sample, and Setting}

The present study employed a parallel randomized controlled trial design, which followed the Consolidated Standards of Reporting Trials (CONSORT, Supplementary File 1) to test the effectiveness of CBT techniques in relieving death anxiety among nurses and eliminating their ageist attitudes towards older adults. A sample of 110 nurses working at a university hospital was selected and recruited using a proportional stratified sampling method. Figure 1 describes the process of recruitment of the participants. Nurses who were caring for older adults and who had been working at the hospital for at least one year were considered eligible. Prior to data collection, written informed consent was obtained from the participants.

\section{Enrollment}

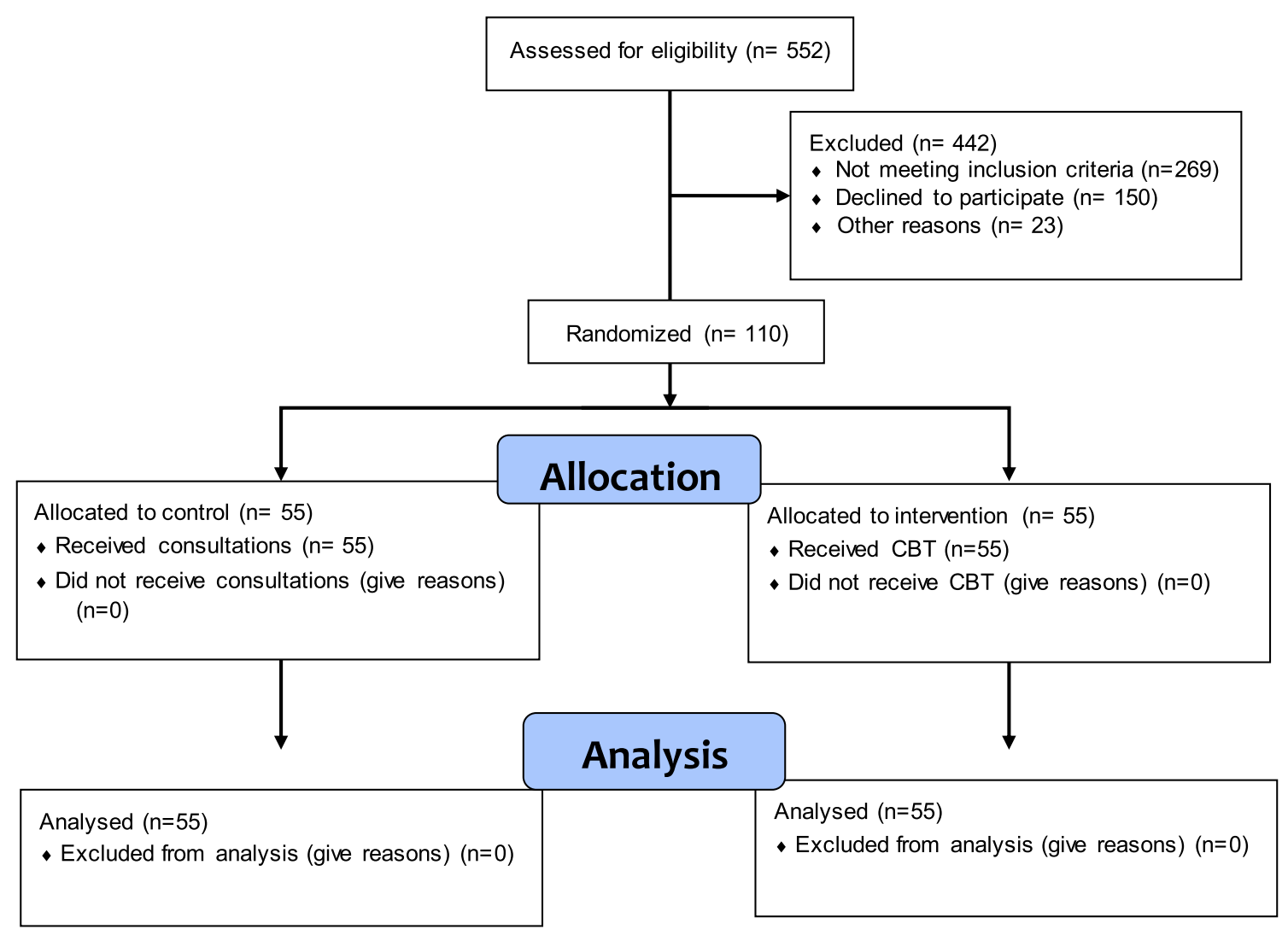

Fig. 1 CONSORT diagram showing participants recruiting and the progress through the trial 


\section{Sample Size}

In total 110 nurses (55 nurses in each group) were recruited in the current study. The sample size was calculated through G*power analysis using the A-priori Sample Size Calculator for MANOVA (Cohen 1988). Given a significance level of $\alpha=0.05$, anticipated large effect size $\left(\eta^{2}=0.14\right)$, a statistical power of 0.95 , this sample size was sufficient for significant statistical analysis.

\section{Ethical Consideration}

IRB approval for this study was obtained from the university and the administrative office of the university hospital. Written informed consent was obtained from all nurses who voluntarily agreed to participate. No personal information was collected from the participants, and all collected data was kept confidential. Further, the researchers assured the participants that participation was voluntary and that they could withdraw from the study at any time without any negative consequences. Participants enrolled in the control group were assured that they would receive the same CBT intervention at the end of the study if the intervention presented positive results on the experimental group. The study protocol was registered by the Protocol Registration and Results System at ClinicalTrial.gov (ID: NCT04319393).

\section{Procedure}

After obtaining the IRB approval and completing the informed consent procedure, the researchers met with the administrator of the hospital to (1) discuss the study purpose and intervention and obtain a list of potential participants, (2) discuss the workflow of the study procedure, and (3) agree on the time/date of site visits for collecting data and running the CBT sessions based on the convenience and work schedules of the nurses. After assessing the potential participants for eligibility, a list of all eligible nurses was recorded on an excel sheet. The researchers then randomly assigned the nurses into control and intervention groups using Excel's RAND function. This function generated a random sequence based on an allocation ratio of 1:1. After randomization was performed, a research assistant distributed the pre-test questionnaires to the nurses in both groups and asked them to drop the completed questionnaires in the designated box next to their work department's front desk. After three days, the research assistant collected the questionnaires.

\section{Intervention}

The CBT intervention was carried out over six two-hour sessions over a period of one month by a well-trained CBT therapist and experienced certified psychologist with a $\mathrm{PhD}$ in Applied Psychology. To enhance the adherence to the intervention protocol, the therapist was trained by experts in CBT and supervised by the researchers. The intervention group was divided into three subgroups of 18 to 19 nurses each. Each subgroup was required to attend two CBT sessions, and the main objective of the CBT intervention was to improve the nurses' self-esteem, interpersonal relationships, and sense of symbolic immortality. An automated text message was sent to the participants daily during the training period to enhance their adherence to the treatment protocol. The message reminded the participants to complete the homework assignments and restudy the training content. Attendance was recorded at the beginning of each session.

The CB therapist followed the same structure in the delivery of each CBT session. In addition to the introductory module, which was aimed at generating the objectives and outcomes of the training sessions, a module of two sessions was developed for each of the study outcomes. Each CBT session was divided into five modules: generating objectives and outcomes, enhancing self-esteem, enhancing interpersonal relationships, changing beliefs regarding symbolic mortality, and changing attitudes regarding death anxiety. Particularly for the purpose of the study, the modules were developed by the CB therapist himself, in collaboration with experts in CBT and the researchers. The modules were based on TMT, cognitive behavioral theories, and previous CBT and psychotherapy programs aimed at improving selfesteem (Kunikata et al. 2016), personal relationships (Song et al. 2018), symbolic immortality (Kissane et al. 2004). These programs were adapted to the context of death anxiety and ageism among nurses. The modules were piloted on 10 nurses and found to be feasible, acceptable, and associated with improved study outcomes. The CBT interventions were guided by the five-factor model, which has been used extensively within the context of CBT (Bagby et al. 2016). A detailed description of the model, which explains the interactions between beliefs, attitudes, emotions, and behaviors, has been presented in recent studies (Bagby et al. 2016; Hawley et al. 2017). The CBT intervention placed emphasis on how nurses can reinforce and enhance their anxiety buffering systems by improving their understanding of how their unconscious realization of their eventual mortality affects their self-reported attitudes. Throughout the intervention, the nurses were trained based on the fivefactor model and therapeutic beliefs, emotions, attitudes, and behaviors pertinent to death anxiety. Further, the differences between therapeutic and nontherapeutic coping strategies of death anxiety were identified and discussed with the nurses in the intervention group.

Based on an analysis of the pre-test questionnaire scores, the CBT intervention was tailored to meet the nurses' training needs, which facilitated the nurses' 
adherence to the treatment protocol. At the beginning of the first session, the objectives of CBT were highlighted. Then, a detailed presentation of the intervention modules was presented to the nurses, with the integration of CBT interventions such as cognitive restructuring, graded exposure, mindfulness meditation, interpersonal skills training, and activity scheduling. A detailed description of the CBT exercises is outlined in Table 1 . To enhance the participants' adherence to the intervention protocol, interactive learning methods, such as group discussions, quizzes, and reflection on real-life stories, were implemented. At the end of the first session, the nurses were assigned a set of homework questions relevant to the CBT exercises. At the beginning of the second session, the participants brainstormed some ideas related to the CBT training assignments from the first session, followed by a discussion of their thoughts regarding the training assignments and reflections on life stories or real experiences of alternative therapeutic behaviors. A checklist of the outcomes and assignments achieved was used by the therapist during each session to ensure the participants' adherence.

The nurses in the control group were divided into the same number of subgroups as the nurses in the intervention group, and they were trained by an experienced gerontological nurse who provided two consultations of $120 \mathrm{~min}$ to each subgroup. The main objectives of the consultations were to (1) instruct the nurses on how to deal with or care for older adults with respect and dignity, taking into account their unique healthcare needs and challenges, and (2) eliminate any ageist attitudes that they might have by targeting myths and misconceptions associated with aging. The control group did not receive active advice or referrals to a psychologist or CBT therapist. During the consultations, the nurses received written and verbal information about the atypical presentation of illness and the most common misconceptions related to aging. The nurses in the control group had the opportunity to discuss and reflect on their own experiences in caring for older adults and in other geriatric care-related issues. The consultations were nurse-centered, goal-oriented, and guided by the nurses' questions. At the end of the final training session, the nurses in both groups (intervention and control) were asked to fill out the post-test questionnaires and return them to the researchers.

\section{Measures}

The outcome variables were measured twice - pre- and post-test. The primary outcomes in the current study were death anxiety and ageism, while the secondary outcomes were self-esteem, interpersonal relationships, and symbolic immortality.

\section{Primary Outcome Measures}

Death Anxiety The revised Collett-Lester Fear of Death Scale (CL-FODS) was used to measure death anxiety (Lester and Abdel-Khalek 2003). The CL-FODS is a 28 -item scale measure of death anxiety which focuses on four main aspects of death and dying: "your own death"; "your own dying"; "the death of others"; and "the dying of others". Each subscale has seven items answered on a 3-point intensity scale (ranging from $1=$ "no" to $5=$ "very"). In the original study, the CL-FODS had very satisfactory reliability scores which ranged from 0.88 to 0.93 , and it had good internal consistency scores which ranged from 0.74 to 0.90 . In the current study, the CL-FODS had satisfactory internal consistency reliability (Cronbach's alpha $=0.79$ ).

Ageism The Fraboni Scale of Ageism (FSA; Fraboni et al. 1990) was used to measure the nurses' ageist attitudes towards older adults. The FSA is a 29-item scale which measures the attitudinal and affective aspects of ageism. The FSA has three positive items (e.g., "Old people can be very creative"), which were reverse coded before calculating the total score. Each item of the FSA has four possible responses: " $1=$ strongly disagree"; " $2=$ disagree"; " $3=$ agree"; and " $4=$ strongly agree". A score range of 29 to 116 was yielded, with higher scores indicating stronger ageist attitudes. In the current study, the FSA had a high internal consistency reliability score (Cronbach's alpha $=0.89$ ) which was very close to that in the original study (0.86) (Fraboni et al. 1990).

\section{Secondary Outcome Measures}

Self-esteem The nurses' self-esteem was measured using the Rosenberg Self-Esteem Scale (RSES; Rosenberg 1989). The RSES has five positive items (e.g., "I take a positive attitude toward myself") and five negative items (e.g., "I feel I do not have much to be proud of"). The nurses responded to these items using a 4-point Likert scale ranging from $1=$ "strongly disagree" to $4=$ "strongly agree". The negative items were reverse coded before calculating the total RSES score. A score range of 10 to 40 was yielded, with higher scores indicating greater levels of self-esteem. The Cronbach's alpha reliability of the RSES in the current study was 0.91 , which is considered high.

Interpersonal Relationships The nurses' interpersonal relationships were measured using the Interpersonal Reactivity Index (IRI; Davis, 1983). The IRI has four subscales, namely perspective-taking, fantasy, empathic concern, and personal distress, with five positive items and two negative items under each subscale. The nurses' responses to each of the 28 items of the IRI ranged from $0=$ "Does not describe me well" to $4=$ "describes me very well". After the negative items were reverse coded, the total IRI score was calculated 


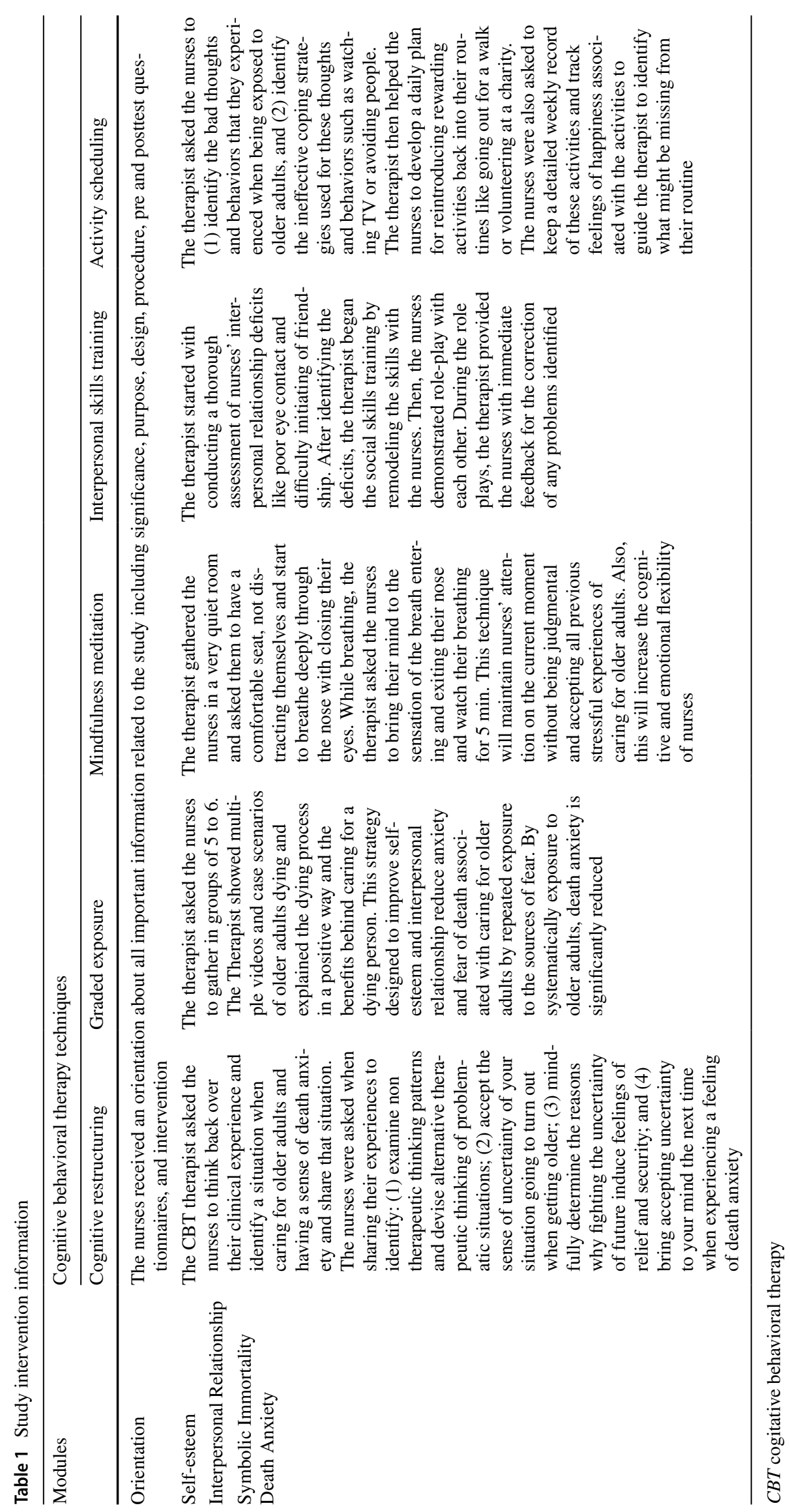


and yielded a range of 0 to 112 . The Cronbach's alpha reliability of the IRI in the current study was 0.81 (i.e. very good).

Symbolic Immortality Symbolic immortality was measured using the Sense of Symbolic Immortality Scale (SSIS) (Drolet 1990). The SSIS contains 11 negative items (e.g., nothing interesting happens in my life) and 15 positive items (e.g., I feel that I am doing what I want in life) about aspects of life related to the desire for symbolic immortality. The nurses responded to these items using a 5-point Likert scale ranging from $1=$ "strongly disagree" to $5=$ "strongly agree". After the negative items were reverse coded, the total score of the SSIS was calculated and yielded a range of 26 to 130 , with higher scores indicating a greater sense of symbolic immortality. The SSIS had good internal consistency in the current study (Cronbach's alpha $=0.86$ ).

\section{Other Measures}

Demographic Questionnaire Some selected demographic characteristics of the nurses, including gender, age, marital status, religion, area of residence, income, level of education and clinical experience, and the number of older adults living in their household, were collected using a demographics questionnaire filled out by the nurses.

\section{Data Analysis}

IBM SPSS Statistics for Windows, Version 25.0. (Armonk, NY: IBM Corp) was used for the analysis of all statistical tests in this study. Descriptive statistics, including mean, standard deviation, and frequency, were used to describe the nurses' levels of self-esteem, interpersonal relationships, sense of symbolic immortality, death anxiety, ageism, sociodemographics, and professional characteristics. Group differences in the baseline data were analysed using chi-square tests (for categorical variables) and t-tests (for continuous variables). A MANOVA was used for comparisons between the intervention and control groups based on the main variables of interest. Also, an individual one-way ANOVA with Bonferroni posthoc test was performed for each dependent variable. ANOVA rather than ANCOVA (with a covariate adjustment for pretest scores) was used because there were no significant differences in the pretest scores between the groups.

\section{Results}

\section{Description of Participants and Study Variables}

Table 2 displays the characteristics of the study participants. A total of 110 nurses agreed to participate in the study and completed the questionnaires. The mean age of the nurses was 35.50 years $(\mathrm{SD}=9.25)$. Among the 110 nurses, $60.9 \%$ $(n=67)$ were male, $55.5 \%(n=61)$ were married, $68.1 \%$ $(\mathrm{n}=75)$ were Muslim, 61.8\% $(\mathrm{n}=68)$ had clinical experience of 5 years or more, $60.9 \%(n=67)$ were working in a medi$\mathrm{cal} /$ surgical unit, $63.6 \%(\mathrm{n}=70)$ were living in rural areas, 65.5\% $(n=72)$ held a bachelor's degree, $56.4 \%(n=62)$ had children, $61.8 \%(n=68)$ had a monthly income of $<500$ JOD, and $91.8 \%(\mathrm{n}=101)$ did not have an older adult living in the same household. There were no significant differences in all sociodemographic and professional characteristics between the intervention and control groups. As illustrated in Table 3, the nurses' levels of symbolic immortality were poor in both the intervention and control groups prior to the training sessions. Meanwhile, prior to the training sessions, the nurses' levels of self-esteem and interpersonal relationships were moderate in both groups, whilst their levels of death anxiety and ageism were high.

\section{Effect of CBT Training}

The findings of the overall MANOVA was statistically significant between the intervention and control group $[\mathrm{F}(5$, $104)=31.69, p<0.001$, partial $\left.\eta^{2}=0.604\right]$. The nurses in the intervention group reported better self-esteem, personal relationship, and symbolic immortality and less death anxiety and ageism than those in the control group. Since the overall MANOVA found to be statistically significant, the individual ANOVAs were analyzed to further test for differences in each dependent variable. The finding of the individual ANOVA was statistically significant for level of self-esteem between the intervention and control groups $\left[\mathrm{F}(1,108)=7.18, \mathrm{p}=0.009\right.$, partial $\left.\eta^{2}=0.062\right]$. The finding of the individual ANOVA was statistically significant for interpersonal relationship between the intervention and control groups $\left[F(1,108)=9.18, p=0.003\right.$, partial $\left.\eta^{2}=0.078\right]$. The finding of the individual ANOVA was statistically significant for symbolic immortality $[\mathrm{F}(1,108)=85.36$, $p<0.001$, partial $\left.\eta^{2}=0.441\right]$. The finding of the individual ANOVA was statistically significant for death anxiety $\left[\mathrm{F}(1,108)=28.52, \mathrm{p}<0.001\right.$, partial $\left.\eta^{2}=0.209\right]$. The finding of the individual ANOVA was statistically significant for ageism $\left[\mathrm{F}(1,108)=24.21, \mathrm{p}<0.001\right.$, partial $\left.\eta^{2}=0.183\right]$. Table 3 shows the results of the overall MANOVA and individual ANOVAs.

\section{Discussion}

Although CBT is well known for targeting psychological distress, this experimental study is the first study to test the effectiveness of CBT in reducing death anxiety and, in turn, ageism among nurses. The present study also investigated 
Table 2 Sociodemographic and professional characteristics of nurses in both groups

\begin{tabular}{|c|c|c|c|c|c|}
\hline \multirow{2}{*}{$\begin{array}{l}\text { Professional/sociodemographic } \\
\text { characteristics }\end{array}$} & \multicolumn{2}{|l|}{ CBT } & \multicolumn{2}{|c|}{ Consultations } & \multirow[t]{2}{*}{$\mathrm{p}$ value } \\
\hline & $\mathrm{N}$ & $\%$ & $\mathrm{~N}$ & $\%$ & \\
\hline Gender & & & & & 0.85 \\
\hline Female & 22 & $40 \%$ & 21 & $39.2 \%$ & \\
\hline Male & 33 & $60 \%$ & 34 & $61.8 \%$ & \\
\hline Marital status & & & & & 0.89 \\
\hline Single & 19 & $34.5 \%$ & 21 & $38.1 \%$ & \\
\hline Married & 31 & $56.4 \%$ & 30 & $54.6 \%$ & \\
\hline Divorced & 5 & $9.1 \%$ & 4 & $7.3 \%$ & \\
\hline \multicolumn{6}{|l|}{ Religion } \\
\hline Muslim & 39 & $70.9 \%$ & 36 & $65.5 \%$ & 0.54 \\
\hline Christian & 16 & $29.1 \%$ & 19 & $34.5 \%$ & \\
\hline \multicolumn{6}{|l|}{ Monthly income } \\
\hline$\geq 500 \mathrm{JOD}$ & 20 & $36.4 \%$ & 22 & $40.0 \%$ & 0.69 \\
\hline$<500 \mathrm{JOD}$ & 35 & $63.6 \%$ & 33 & $60.0 \%$ & \\
\hline \multicolumn{6}{|l|}{ Area of residence } \\
\hline Urban & 21 & $38.1 \%$ & 19 & $34.5 \%$ & 0.69 \\
\hline Rural & 34 & $61.9 \%$ & 36 & $65.5 \%$ & \\
\hline \multicolumn{6}{|l|}{ Level of education } \\
\hline BSN & 37 & $67.3 \%$ & 35 & $63.6 \%$ & 0.68 \\
\hline MSN & 18 & $32.7 \%$ & 20 & $36.4 \%$ & \\
\hline Having children & & & & & 0.70 \\
\hline Yes & 30 & $54.6 \%$ & 32 & $58.2 \%$ & \\
\hline No & 25 & $45.4 \%$ & 23 & $41.8 \%$ & \\
\hline Year of experience & & & & & 0.84 \\
\hline$\geq 5$ years & 37 & $67.3 \%$ & 36 & $65.5 \%$ & \\
\hline$<5$ years & 18 & $32.7 \%$ & 19 & $34.5 \%$ & \\
\hline Working department & & & & & 0.61 \\
\hline Surgical/medical & 32 & $58.2 \%$ & 35 & $63.6 \%$ & \\
\hline $\mathrm{ICC} / \mathrm{CCU}$ & 12 & $21.8 \%$ & 8 & $14.6 \%$ & \\
\hline Emergency & 11 & $20 \%$ & 12 & $21.8 \%$ & \\
\hline $\begin{array}{l}\text { Having an older adult in the same } \\
\text { household }\end{array}$ & & & & & 0.73 \\
\hline Yes & 5 & $9.1 \%$ & 4 & $7.3 \%$ & \\
\hline \multirow[t]{2}{*}{ No } & 50 & $90.9 \%$ & 51 & $92.7 \%$ & \\
\hline & Mean & SD & Mean & SD & $\mathrm{p}$ value \\
\hline Age & 37.80 & 8.55 & 33.30 & 10.15 & 0.13 \\
\hline Year of experience & 11.85 & 8.16 & 13.05 & 8.96 & 0.46 \\
\hline
\end{tabular}

CBT cognitive behavioral therapy, ICC/CCU Intensive Care Unit/Critical Care Unit

the impact of the use of CBT techniques on nurses' selfesteem, interpersonal relationships, and sense of symbolic immortality. The use of CBT techniques was found to enhance the three components of nurses' anxiety buffering systems, which led to lower levels of death anxiety and ageism among the nurses.

The findings showed that, in comparison to the nurses in the control group, the nurses who received CBT training had significantly improved levels of self-esteem and interpersonal relationships and a greater sense of symbolic immortality. The impact of CBT on self-esteem has been evidenced in previous studies conducted on different populations. For example, Easvaradoss and Cabral (2015) found CBT to improve self-esteem levels among nursing students, and similar studies have supported these findings (Edward et al. 2015; Tsai et al. 2019). Other studies have found CBT effective in improving the self-esteem of participants with several conditions, including drug abuse (Hamidipour et al. 
Table 3 One way multivariate and univariate ANOVA of posttest of intervention and control groups $(\mathrm{N}=110)$

\begin{tabular}{lllrrr}
\hline Variables & $\begin{array}{l}\text { Control group } \\
\text { M (SD) }\end{array}$ & $\begin{array}{l}\text { Intervention Group } \\
\text { M (SD) }\end{array}$ & F & $\mathrm{p}$ & Partial $\eta^{2}$ \\
\hline Combined effect & & & 31.69 & $<0.001$ & 0.604 \\
Self-esteem & $33.2(1.11)$ & $34(1.92)$ & 7.18 & 0.009 & 0.062 \\
Interpersonal relationship & $93.32(3.07)$ & $95.12(3.16)$ & 9.18 & 0.003 & 0.078 \\
Symbolic immortality & $59.12(2.12)$ & $62.66(2.70)$ & 85.36 & $<0.001$ & 0.441 \\
Death anxiety & $67.65(7.72)$ & $60.46(6.32)$ & 28.52 & $<0.001$ & 0.209 \\
Ageism & $68.11(7.42)$ & $60.76(8.22)$ & 24.21 & $<0.001$ & 0.183 \\
\hline
\end{tabular}

ANOVA analysis of variances, $M$ mean, $S D$ standard deviation
2018), depression (Alavi et al. 2017), and other health problems (Sadler et al. 2018). However, the current study is the first to report findings on the effectiveness of CBT in improving the self-esteem levels of nurses and hence reducing their ageism towards older adults.

The mechanisms of CBT and its relation to self-esteem were described in Fennell's conceptualization model, which explains that people with long-term anxiety develop negative judgments about themselves, especially in their early life (Fennell et al. 2010). The negative perceptions that nurses have towards caring for older adults are considered disruptive emotions which contribute to the development of ageist attitudes and low self-esteem among nurses (Greenberg et al. 2017). CBT highlights the interrelation between disruptive emotions and attitudes and provides interventions tailored to the context of persistent disruptive beliefs about the self (Geller and Dunn 2011). Further, CBT acts to modify the ways nurses perceive and interact with the stress associated with ageism and death anxiety. As this was the first study to explore the effect of CBT on nurses' self-esteem, further studies are needed to evaluate the role of CBT intervention on nurses' self-esteem and mental health.

The use of CBT techniques also proved to have a significant positive effect on nurses' interpersonal relationships. Death anxiety is associated with increased psychological strain (Zhuo et al. 2016) and social and role dysfunction that negatively impact interpersonal relationships (Craighead and Dunlop 2014). The high levels of anxiety associated with caring for older adults may lead nurses to avoid interacting or spending time with older adults (Deasey et al. 2018) or, in some cases, to address them in an offensive or disrespectful manner (Liu et al. 2013, 2015). CBT has been reported to enhance people's empathy measures and positive emotions and attitudes towards older adults. Further, CBT helps in monitoring and regulating emotional processes through emotional resonance (Balconi and Vanutelli 2017). Also, CBT emphasizes positive interactions and therefore improves the autonomic responsiveness underlying the human ability to perceive and empathize with one's own emotions and the emotions of others (Balconi and Vanutelli 2017). Incorporating CBT principles such as psychological interventions, interpersonal communication, and teamwork skills in the training programs of beginner nurses is associated with improved interpersonal relationships between nurses and their patients (Butler et al. 2014; Yoshinaga et al. 2015).

The application of CBT techniques was also found to enhance the nurses' sense of symbolic immortality, which is the third component of the anxiety buffering system. CBT training provides nurses with a sense of symbolic immortality by increasing their awareness of and engagement in adaptive behaviors required for alleviating death anxiety, eliminating death thoughts, and underestimating vulnerability to death (Williams 2017). In the study of Roberts and Maxfield (2019), CBT motivated participants to actively engage in prosocial and charitable activities with older adults, allowing them to better understand their shared conception of death reality and manage their death anxiety. In the current study, nurses who received CBT training reacted positively to the concept of mortality salience. The nurses came to accept the idea of death and countered death awareness by showing increased acceptance of and support for others, seeking comfort in caring for vulnerable people, and reducing their in-group bias.

The enhancement of the three components of the nurses' anxiety buffering systems led to significant improvements in the death anxiety levels and ageist attitudes of the nurses in the intervention group compared to the nurses in the control group. This finding is similar to the findings of similar recent studies conducted among different populations (Freedland et al. 2015; Dekker et al. 2012; Gary et al. 2010). For example, in a study by Freedland et al. (2015), CBT was found to be associated with improved health-related quality of life, reduced anxiety, increased emotional engagement, enhanced nurse-patient mutual respect, and improved social functioning among patients undergoing hemodialysis compared to patients receiving routine care. Further, different CBT interventions, including assertiveness training, self-control therapy, problem-solving skills training, and relaxation training, have been associated with improved quality of care and reduced depression and strain in people with musculoskeletal disorders (Castro et al. 2012). 
It is suggested that CBT empowers nurses to effectively manage and handle their death anxiety and its associated ageism as long as they adhere to the rules for living. When providing healthcare to older adults, nurses may overlook the 'rules for living' (Stokoe 2015). Consequently, the disruptive belief of mortality salience is stimulated, and subsequent destructive cycles of detrimental feelings (death anxiety) and negative attitudes and behaviors (ageism), which maintain and exacerbate disruptive beliefs, are activated (Ben-Harush et al. 2017). These maladaptive dysfunctional cognitive and behavioral responses expressed by nurses caring for older adults can be easily targeted with CBT (Ben-Harush et al. 2017), as CBT focuses on identifying and changing the function, content, and structure of cognition associated with these dysfunctional behavioral responses (Hoffart et al. 2018). In a recent study, CBT was shown to help nurses learn alternative methods of thinking or behavior modification (Tanoue et al. 2018). CBT identifies problems and boosts positive thinking through the modification of dysfunctional emotions, behaviors, and thoughts. These findings are consistent with those of our study, which have shown the use of CBT techniques to be effective in reducing death anxiety and ageism among nurses.

\section{Relevance to Clinical Practice}

Targeting ageism in healthcare settings requires multidisciplinary collaboration between governmental agencies and healthcare professionals. Health policymakers need to raise the awareness of governments regarding the importance of funding research and projects in geriatric care and ageism. The study findings could aid hospital managers in developing ongoing CBT and geriatric care for older adults training for their nursing staff. In this regard, several specific recommendations can be made for achieving optimal geriatric care and psychological health of nurses. Ongoing training programs which focus on caring for older adults and the process of aging should be tailored to both the learning needs of nurses and the unmet needs of older adults. Further, by incorporating CBT interventions into these training programs, hospital managers may start to develop a vision for supporting their nursing staff in pursuing academic research careers in the psychogeriatric field. Emerging research programs of psychological interventions like CBT will empower nurses with the professional education and development necessary for overcoming the barriers to providing older adults with good quality healthcare.

\section{Limitations}

Although the present study has identified a gap in the nursing literature and has shed more light on the value of CBT in reducing death anxiety and ageism among nurses caring for older adults, it is not without limitations. Whilst the recruited subjects were representative of the study population, the limited number of recruited nurses may affect the generalizability of the findings. Further, self-esteem, interpersonal relationships, and symbolic immortality are socio-culturally driven and context-dependent constructs that may have substantially different indicators in other societies. According to Sandlund et al. (2018), blinding the study participants can minimize the bias associated with the variations (differences) between the control and intervention groups. However, in the present study, the participants were not blinded to the treatment assignment, as it is difficult to blind participants to psychological interventions (Sandlund et al. 2018). Moreover, the current exceptional circumstances imposed by the COVID-19 pandemic and lockdown have made it significantly challenging to evaluate the results six months after the intervention, due to the limited availability of the nurses and their intensive workloads. Therefore, this paper reports only on the pre-test and posttest measurements of the study variables, with no follow-up data. The use of the self-report questionnaires to collect data may have increased the possibility of recall bias, which may limit the generalizability of the findings. This study may also have suffered from the "Hawthorne Effect", whereby the nurses may have provided inaccurate responses due to them being aware that they were participating in the study.

\section{Conclusion}

Our findings have shown that CBT intervention significantly reduces ageism outcomes related to death anxiety, low self-esteem, poor interpersonal relationships, and disruptive sense of symbolic immortality. This study suggests that cost-effective interventions like CBT should be incorporated in the orientation training programs for nurses caring for older adults to eliminate any ageist attitudes or behaviors the nurses may have. Further investigation using a well-controlled intervention study design on a larger sample is strongly recommended for future research. A future follow-up research study which reassesses the study outcomes at a 12-month interval is recommended to provide further evidence about the effectiveness of CBT. Future research which use a psychological-based intervention as a control treatment is recommended. Future studies which investigate the effectiveness of CBT on other forms of discrimination, such as racism and sexism, in healthcare settings are also recommended.

Acknowledgements We want to thank the faculty of Scientific Research at Jordan University of Science and Technology for providing us with the required logistic and financial support throughout the study procedure. 
Funding This study was funded by Jordan University of Science and Technology [Grant Number 21702018].

\section{Compliance with Ethical Standards}

Conflict of Interest Mohammad Rababa, Hossam Alhawatmeh, Nahla Al Ali, and Manal Kassab have declare that there are no conflicts of interest.

Informed Consent Informed consent was obtained from all individual participants included in the study.

Animal Rights No animal studies were carried out by the authors for this article.

\section{References}

Alavi, M., Molavi, H., \& Molavi, R. (2017). The impact of cognitive behavioral therapy on self-esteem and quality of life of hospitalized amputee elderly patients. Nursing and Midwifery Studies, 6(4), 162-167.

Bagby, R. M., Gralnick, T. M., Al-Dajani, N., \& Uliaszek, A. A. (2016). The role of the five-factor model in personality assessment and treatment planning. Clinical Psychology: Science and Practice, 23(4), 365-381.

Balconi, M., \& Vanutelli, M. E. M. E. (2017). Empathy in negative and positive interpersonal interactions. What is the relationship between central (EEG, fNIRS) and peripheral (autonomic) neurophysiological responses? Advances in Cognitive Psychology, 13(1), 105-120.

Barnett, M. D., \& Adams, C. M. (2018). Ageism and aging anxiety among young adults: Relationships with contact, knowledge, fear of death, and optimism. Educational Gerontology, 44(11), 693-700.

Ben-Harush, A., Shiovitz-Ezra, S., Doron, I., Alon, S., Leibovitz, A., Golander, H., et al. (2017). Ageism among physicians, nurses, and social workers: Findings from a qualitative study. European Journal of Ageing, 14(1), 39-48.

Bergman, Y. S., Bodner, E., \& Shrira, A. (2018). Subjective nearness to death and end-of-life anxieties: The moderating role of ageism. Aging \& Mental Health, 22(5), 678-685.

Butler, M. P., Begley, M., Parahoo, K., \& Finn, S. (2014). Getting psychosocial interventions into mental health nursing practice: A survey of skill use and perceived benefits to service users. Journal of Advanced Nursing, 70(4), 866-877.

Castro, M. M. C., Daltro, C., Kraychete, D. C., \& Lopes, J. (2012). The cognitive behavioral therapy causes an improvement in quality of life in patients with chronic musculoskeletal pain. Arquivos de NeuroPsiquiatria. Scielo, 70(11), 864-868. https:// doi.org/10.1590/S0004-282X2012001100008

Chang, E.-S., Kannoth, S., Levy, S., Wang, S.-Y., Lee, J. E., \& Levy, B. R. (2020). Global reach of ageism on older persons' health: A systematic review. PLoS ONE, 15(1), e0220857.

Cohen, J. (1988). Statistical power analysis Jbr the behavioral. Sciences (pp. 18-74). Hillsdale (NJ): Lawrence Erlbaum Associates.

Cohen-Mansfield, J., Golander, H., Iecovich, E., \& Jensen, B. (2019). Social engagement care for frail older persons: Desire for it and provision by live-in migrant caregivers. The Journals of Gerontology: Series B, 74(6), 1062-1071.

Craighead, W. E., \& Dunlop, B. W. (2014). Combination psychotherapy and antidepressant medication treatment for depression:
For whom, when, and how. Annual Review of Psychology, 65, 267-300.

Davis, M. H. (1983). Measuring individual differences in empathy: Evidence for a multidimensional approach. Journal of Personality and Social Psychology, 44, 113-126.

Deasey, D., Kable, A., \& Jeong, S. (2018). An exploration of emergency nurses' understanding of the ageing process and knowledge of their older patient: A comparison between regional and metropolitan nurses in Australia. International Emergency Nursing, 37, 44-51.

Dekker, R. L., Moser, D. K., Peden, A. R., \& Lennie, T. A. (2012). Cognitive therapy improves three-month outcomes in hospitalized patients with heart failure. Journal of Cardiac Failure, 18(1), 10-20.

Dobson, D., \& Dobson, K. S. (2018). Evidence-based practice of cognitive-behavioral therapy. New York: Guilford Publications.

Drolet, J. L. (1990). Transcending death during early adulthood: Symbolic immortality, death anxiety, and purpose in life. Journal of Clinical Psychology, 46(2), 148-160.

Easvaradoss, V., \& Cabral, V. (2015). Impact of cognitive behavior group therapy on the psychological functioning of adolescents from dual earner families. Indian Journal of Health \& Wellbeing, 6(2), 177-180.

Edward, K. L., Warelow, P., Hemingway, S., Hercelinskyj, G., Welch, A., McAndrew, S., \& Stephenson, J. (2015). Motivations of nursing students regarding their educational preparation for mental health nursing in Australia and the United Kingdom: A survey evaluation. BMC Nursing, 14(1), 29.

Fennell, M. L., Prabhu Das, I., Clauser, S., Petrelli, N., \& Salner, A. (2010). The organization of multidisciplinary care teams: Modeling internal and external influences on cancer care quality. Journal of The National Cancer Institute Monographs, 2010(40), 72-80.

Fialová, D., Kummer, I., Držaić, M., \& Leppee, M. (2018). Ageism in medication use in older patients. In L. Ayalon \& C. TeschRömer (Eds.), Contemporary perspectives on ageism (pp. 213240). Cham: Springer.

Fraboni, M., Saltstone, R., \& Hughes, S. (1990). The Fraboni Scale of Ageism (FSA): An attempt at a more precise measure of ageism. Canadian Journal on Aging/La revue canadienne du vieillissement, 9(1), 56-66.

Freedland, K. E., Carney, R. M., Rich, M. W., Steinmeyer, B. C., \& Rubin, E. H. (2015). Cognitive behavior therapy for depression and self-care in heart failure patients: A randomized clinical trial. JAMA Internal Medicine, 175(11), 1773-1782.

Gary, R. A., Dunbar, S. B., Higgins, M. K., Musselman, D. L., \& Smith, A. L. (2010). Combined exercise and cognitive behavioral therapy improves outcomes in patients with heart failure. Journal of Psychosomatic Research, 69(2), 119-131.

Geller, J., \& Dunn, E. C. (2011). Integrating motivational interviewing and cognitive behavioral therapy in the treatment of eating disorders: Tailoring interventions to patient readiness for change. Cognitive and Behavioral Practice, 18(1), 5-15.

Gipe, K., \& Kerstein, S. J. (2018). Let us be fair to 5-year-olds: Priority for the young in the allocation of scarce health resources. Public Health Ethics, 11(3), 325-335.

Glendenning, F. (2018). Educational gerontology: International perspectives. London: Routledge.

Greenberg, J., Helm, P., Maxfield, M., \& Schimel, J. (2017). How our mortal fate contributes to ageism: A terror management perspective. In T. D. Nelson (Ed.), Ageism: Stereotyping and prejudice against older persons (pp. 105-132). London: MIT Press.

Hamidipour, R., Heydari, H., Bahari, F., \& Taghavi, M. (2018). Effectiveness of group cognitive behavioral counseling on academic self-efficacy and sense of belonging to school in 
students with academic failure. Community Health Journal, 12(1), 69-78.

Hawley, L. L., Rogojanski, J., Vorstenbosch, V., Quilty, L. C., Laposa, J. M., \& Rector, N. A. (2017). The structure, correlates, and treatment related changes of mindfulness facets across the anxiety disorders and obsessive compulsive disorder. Journal of Anxiety Disorders, 49, 65-75.

Helm, P. J., Duchschere, J. E., \& Greenberg, J. (2018). Treating low self-esteem: Cognitive behavioural therapies and terror management theory. Curing the Dread of Death: Theory, Research and Practice, 16, 219-237.

Hoffart, A., Johnson, S. U., Nordahl, H. M., \& Wells, A. (2018). Mechanisms of change in metacognitive and cognitive behavioral therapy for treatment-resistant anxiety: The role of metacognitive beliefs and coping strategies. Journal of Experimental Psychopathology, 9(3), 2043808718787414.

Kessler, E.-M., Tempel, J., \& Wahl, H.-W. (2014). Concerns about one's aging: The role of work context and psychological distress. GeroPsych - The Journal of Gerontopsychology and Geriatric Psychiatry, 27, 81-86.

Kissane, D. W., Grabsch, B., Clarke, D. M., Christie, G., Clifton, D., Gold, S., et al. (2004). Supportive-expressive group therapy: The transformation of existential ambivalence into creative living while enhancing adherence to anti-cancer therapies. PsychoOncology, 13(11), 755-768.

Krütli, P., Rosemann, T., Törnblom, K. Y., \& Smieszek, T. (2016). How to fairly allocate scarce medical resources: Ethical argumentation under scrutiny by health professionals and lay people. PLOS ONE, 11(7), e0159086.

Kunikata, H., Yoshinaga, N., \& Nakajima, K. (2016). Effect of cognitive behavioral group therapy for recovery of self-esteem on community-living individuals with mental illness: Non-randomized controlled trial. Psychiatry and Clinical Neurosciences, 70(10), $457-468$.

Lester, D., \& Abdel-Khalek, A. (2003). The Collett-Lester fear of death scale: A correction. Death Studies, 27(1), 81-85.

Levy, S. R. (2018). Toward reducing ageism: PEACE (positive education about aging and contact experiences) model. The Gerontologist, 58(2), 226-232.

Liu, Y. E., Norman, I. J., \& While, A. E. (2013). Nurses' attitudes towards older people: A systematic review. International Journal of Nursing Studies, 50(9), 1271-1282.

Liu, Y. E., Norman, I. J., \& While, A. E. (2015). Nurses' attitudes towards older people and working with older patients: An explanatory model. Journal of Nursing Management, 23(8), 965-973.

Menzies, R. E., \& Dar-Nimrod, I. (2017). Death anxiety and its relationship with obsessive-compulsive disorder. Journal of Abnormal Psychology, 126(4), 367-377.

Rababa, M., Hammouri, A. M., Hweidi, I. M., \& Ellis, J. L. (2020). Association of nurses' level of knowledge and attitudes to ageism toward older adults: Cross-sectional study. Nursing \& Health Sciences, 22(3), 593-601. https://doi.org/10.1111/nhs.12701

Roberts, J. R., \& Maxfield, M. (2019). Mortality salience and age effects on charitable donations. American Behavioral Scientist, 63(14), 1863-1884

Rosenberg, M. (1989). Society and the adolescent self-image (Rev). Middeltown, CT: Wesleyan University Press.

Sadler, P., McLaren, S., Klein, B., \& Jenkins, M. (2018). Advancing cognitive behaviour therapy for older adults with comorbid insomnia and depression. Cognitive Behaviour Therapy, 47(2), 139-154.

Salter, B., \& Salter, C. (2018). The politics of ageing: Health consumers, markets and hegemonic challenge. Sociology of Health \& Illness, 40(6), 1069-1086.

Sandlund, C., Hetta, J., Nilsson, G. H., Ekstedt, M., \& Westman, J. (2018). Impact of group treatment for insomnia on daytime symptomatology: Analyses from a randomized controlled trial in primary care. International Journal of Nursing Studies, 85, 126-135.

São José, J. M. S., Amado, C. A. F., Ilinca, S., Buttigieg, S. C., \& Taghizadeh Larsson, A. (2019). Ageism in health care: A systematic review of operational definitions and inductive conceptualizations. The Gerontologist, 59(2), e98-e108.

Shiovitz-Ezra, S., Ayalon, L., Brodsky, J., \& Doron, I. I. (2016). Measuring ageism based on knowledge, attitudes and behavior: Findings from an Israeli pilot study. Ageing International, 41(3), 298-310.

Song, M.-K., Choi, S.-H., Lee, D.-H., Lee, K.-J., Lee, W. J., \& Kang, D.-H. (2018). Effects of cognitive-behavioral therapy on empathy in patients with chronic pain. Psychiatry Investigation, 15(3), $285-291$.

Stokoe, N. (2015). Youth is wasted on the young, therapy is wasted on the old? A discussion on the attitudes, challenges and considerations of working psychologically with older adults. Clinical Psychology, 276, 36-53.

Tanoue, H., Yoshinaga, N., Kato, S., Naono-Nagatomo, K., Ishida, Y., \& Shiraishi, Y. (2018). Nurse-led group cognitive behavioral therapy for major depressive disorder among adults in Japan: A preliminary single-group study. International Journal of Nursing Sciences, 5(3), 218-222.

Tsai, M. C., Shen, P. D., Chen, W. Y., Hsu, L. C., \& Tsai, C. W. (2019). Exploring the effects of web-mediated activity-based learning and meaningful learning on improving students' learning effects, learning engagement, and academic motivation. Universal Access in the Information Society, 1-16.

Williams, M. J. (2017). Prosocial behavior following immortality priming: Experimental tests of factors with implications for CVE interventions. Behavioral Sciences of Terrorism and Political Aggression, 9(3), 153-190.

Wyman, M. F., Shiovitz-Ezra, S., \& Bengel, J. (2018). Ageism in the health care system: Providers, patients, and systems. In L. Ayalon \& C. Tesch-Römer (Eds.), Contemporary perspectives on ageism (pp. 193-212). Cham: Springer.

Yoshinaga, N., Nosaki, A., Hayashi, Y., Tanoue, H., Shimizu, E., Kunikata, H., et al. (2015). Cognitive behavioral therapy in psychiatric nursing in Japan. Nursing Research and Practice, 2015, 529107. https://doi.org/10.1155/2015/529107

Zhuo, C. J., Bian, H. M., Gao, Y. J., Ma, X. L., Ji, S. Z., Yao, M. Y., \& Li, G. Y. (2016). Nonspecific effect of stress on brain gray matter volume in drug-naive female patients with first depressive episode. Chinese Medical Journal, 129(3), 279-283.

Publisher's Note Springer Nature remains neutral with regard to jurisdictional claims in published maps and institutional affiliations. 